Brown \& Ferguson - Beat Machine, revised. 13 April 2020.

\title{
Beat Machine:
}

\section{Embracing the creative limitations and opportunities of low-cost computers}

Andrew R. Brown (artist, researcher, educator), Queensland College of Art, Griffith University, 226 Grey St, South Brisbane, QLD 4101, Australia. Email:

$<$ andrew.r.brown@griffith.edu.au>. Website: <andrewrbrown.net.au>. ORCID: 0000-00034102-8301.

John R. Ferguson (artist, researcher, educator), Queensland Conservatorium Griffith University, 140 Grey St, South Brisbane, QLD 4101, Australia. Email:

<john.ferguson@griffith.edu.au>.Website: <johnrobertferguson.com>. ORCID: 0000-00029274-6795.

\section{Abstract}

The Beat Machine is a hand-held music synthesizer and sequencer. We discuss the development of the Beat Machine and how creative constraints and opportunities were introduced by the particularities of low-cost microprocessors and associated electronics. The discussion is framed as an exemplar of Kåre Poulsgaard's concept of enactive individuation, a framework for relating material engagement to digital design and fabrication. In reflecting on the design and making of the Beat Machine we connect this framework with more established notions of creative interaction and the affordances of digital media.

\section{Genesis}

The genesis for the project can be traced to Handmade Electronic Music by Nic Collins, specifically 'The World's Simplest Circuit: Six Oscillators on a Chip, Guaranteed to Work' [1] p.129. A battery powered synthesizer based around a printed circuit board (PCB) and a 40106 (Hex Schmitt-Trigger) with audio control via photocells, touch-pads, and potentiometers was designed by John Ferguson in 2018 [2] but a desire to include algorithmic 
Brown \& Ferguson - Beat Machine, revised. 13 April 2020.

coding in the design to add sonic flexibility led to a collaboration with Andrew R. Brown; the Beat Machine was born. Like many designs, the Beat Machine has been through several iterations with versions having been used for musical performances in the Creative Music Technology program at Griffith University, Brisbane, Australia.

\section{Background}

The Beat Machine is an electronic musical instrument that produces sound through synthesis and arranges these sounds in looped patterns using a multitrack step sequencer. Video documentation of the Beat Machine can be found online [3]. The Beat Machine aligns with popular electronic music practices using synthesizers, sequencers and drum machines dating from the mid 20th century and repopularized in the early 21 st century. These instruments have established themselves as part of Western musical culture and DIY versions such as the Beat Machine provide an accessible path into this culture whilst serving to reveal some technical underpinnings often obscured by commercial tools.

The Beat Machine is designed to be distributed as a DIY kit for musicians. Users are assumed to be inexperienced builders of electronic devices and so the assembly process is hoped to be educational. Component costs are kept low to maximise access and minimise the impact of assembly errors. The resulting lo-fi audio output is embraced as part of the Beat Machine's character. It is anticipated that users are interested in popular electronic music which the Beat Machine is designed to produce. It is designed to cater to users with modest musical experience. Importantly, the Beat Machine is a platform that can be extended by users, especially on the software front. For example, it has been repurposed as a MIDI controller when connected via USB to another computer and, like all Arduino-class devices, can be reprogrammed by users to perform differently. This extensibility of DIY resources reinforces the educational agenda of the designers.

Despite its modest materials, the Beat Machine is still intended to be an effective live musical instrument. Reflecting the designers' interest in live electronic music performance, the aim was to achieve a fluid interaction during performance, and efficient interaction during editing. The user experience ideally resembles a seamless performative experience that is described variously as "flow" [4] "meaningful engagement" [5] or "musical homeostasis" [6] 
Brown \& Ferguson - Beat Machine, revised. 13 April 2020.

All three terms, interestingly, arise from research into the phenomenology of creative arts practice and the latter term is specific to musical human-technology interactions.

While the Beat Machine may have some unique combination of features to differentiate it from related projects, this article is not so much about the project's uniqueness but rather about exploring the development of the Beat Machine as a case study in the computational design of post-digital DIY musical instruments [7, 8]. In particular, we examine our experience as posthumanist designers, makers, and users through the lens of enactive individuation [9].

\section{Enactive Individuation}

The concept of enactive individuation, formulated by Kåre Poulsgaard, describes how mind and form are emergent properties of the dynamic integration of technology, memory, and aesthetics. It extends the frameworks of material engagement theory developed by Lambros Malafouris [10] into the realm of digital creative work.

"Enactive individuation seeks to describe how ongoing -- affective -- transactions between mind, materials, and information logics extend imaginative capacities for computational design thinking." [11]

An enactive perspective assumes that affect arises from participation. For humans, this means that embodied involvement and contextual influence -- not simply intellectual attention -- are crucial aspects of engagement in activities and experience. For non-human actors such as physical objects, ideas and contexts, this means that their presence and characteristics are acknowledged as influential in shaping activities and outcomes. Projects like the Beat Machine also manifest an enactive perspective on computational design by reinforcing the significance of prototyping.

The concept of individuation draws on Bernard Stiegler's [12] phenomenology of technics and time to interpret the transformative relationship between technology and cognition. In this view, the individual characteristics of people and things are shaped by, and emerge from, the dynamics of their interactions. Whilst it is widely accepted that people's individuality is shaped by their interactions with the world, for theorists like Gilbert Simondon [13] the 
Brown \& Ferguson - Beat Machine, revised. 13 April 2020.

creation of forms transcends mere association of parts to become acts of individuation. Thus, the creation of the Beat Machine is a process of individuation.

A key to the power of these transformative relationships with technology is the way in which structures of knowledge and materiality are recursively built and layered. It has long been recognised that technologies externalise knowledge, technique, and purpose. In this way software algorithms reflect a programmer's ideas, and hardware systems reveal the priorities and perspectives of their makers. Prominent philosophical advocates of this position include Martin Heidegger whose concept of thrownness suggests that technologies, far from being neutral actors, propel users toward particular actions [14]. Bernard Stiegler, following Jacques Derrida, emphasises the tendency for technologies to grammatize, by which he means that technics formalise processes and patterns of behaviour and thought (just as grammar structures language) resulting in both ongoing stability and immobility [15]. Such stability is a necessary part of arriving at an individualised form.

Our experiences in the making of the Beat Machine seem to resonate with the concept of enactive individuation. Even though we were not fully conscious of this perspective during the Beat Machine's development, the concept is revealing as a backdrop for our description of product design and making.

\section{Inside the Beat Machine}

Developing the Beat Machine involved a creative and exploratory practice, in which thinking and doing became a unified process. An important aspect was selecting different tools, platforms and metaphors which each recast design problems because each afforded differing solutions and understandings. Our processes of design and making broadly align with how Poulsgaard describes the digital development processes he observed, concluding that;

"Computational design does not arise from manipulation of mental plans and representations, but rather from within the complex interplay of forces in a given ecology reaching across material and informational domains." [16]

To structure our discussion about the development of the Beat Machine, we use aspects of interaction design outlined by Jung et al. which, they suggest, should help us "examine 
Brown \& Ferguson - Beat Machine, revised. 13 April 2020.

multifaceted roles of metaphors and materialities." [17] p.1. These aspects are: Surface, the user interface; Behaviour, re

\section{Surface}

Interface design requires bringing elements together into a coherent whole. The first example of individuation in the Beat Machine project is its interface design, which adopts a DIY aesthetic that embraces raw exposed components and unadorned controls. The identity of the Beat Machine as a form relies on establishing its particular interface (see Figure 1). In recent years DIY aesthetics have been adopted by commercial music products such as the Pocket Operators by Teenage Engineering.

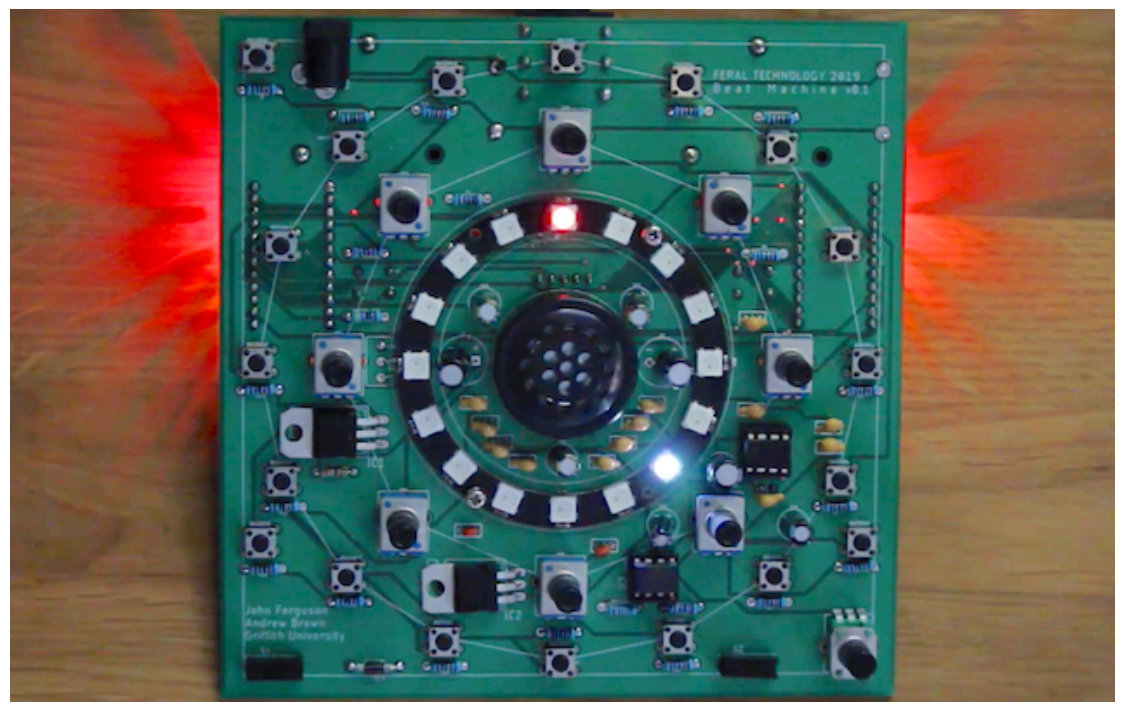

Fig. 1: Beat Machine v1 top view.

The circular arrangement of components reinforces the looping structure of the sequencer engine. These visual metaphors are particularly necessary in digital devices because software-based patterns are otherwise opaque to users. Beat Machine interface design pays attention to this and other principles of digital design outlined by Jung and colleagues who suggest that;

"The forms of interactive [digital] applications are more complex than those of analog objects due to the immaterial qualities of digital materials, the temporal nature of their coded behaviours, interaction possibilities transcending physical material properties, and their network connectivity to other digital artists." [18] 
Brown \& Ferguson - Beat Machine, revised. 13 April 2020.

The size of the device is partly dictated by component spacing requirements, but also designed to be easily handheld, allowing for expressive gestural performances. An accelerometer on the back is used to map device tilt orientation onto sonic features. Thus, the individual identity of the Beat Machine arises from the interaction of the materiality of components and the embodied requirements of hand-held musical performance.

\section{Behaviour}

The Beat Machine has three major software components: synthesis engine, sequencer engine, and interface management.

The synthesis architecture aimed at reproducing percussive and pitched timbres similar to drum machines such as the Roland TR-808. Requirements of the low cost microprocessors utilised in the design demanded very efficient software. Fortunately, the Mozzi audio library [19] provided an excellent platform for this project and its selection also influenced the choice of Arduino microprocessors. The agency of these material choices on the development of the project is clearly evident in such decisions.

The synthesis architecture of the Beat Machine is shown in Figure 2. Each of the three voices has two oscillators mixed into an attack-release amplitude envelope with volume controls for per-voice and overall output. The 8 bit, 16khz audio output from the Mozzi library dictates a lo-fi aesthetic and listening is therefore a particularly critical aspect of the design processes. Theoretical signal processing expectations were useful only as a guide, and embodied senses were always the final arbiter of 'best' choices, reinforcing Poulsgaard's view that "Material engagement acts as a fundamental cognitive resource in its own right -that we think with and through things in action." [20] 
Brown \& Ferguson - Beat Machine, revised. 13 April 2020.

\section{Beat Machine}

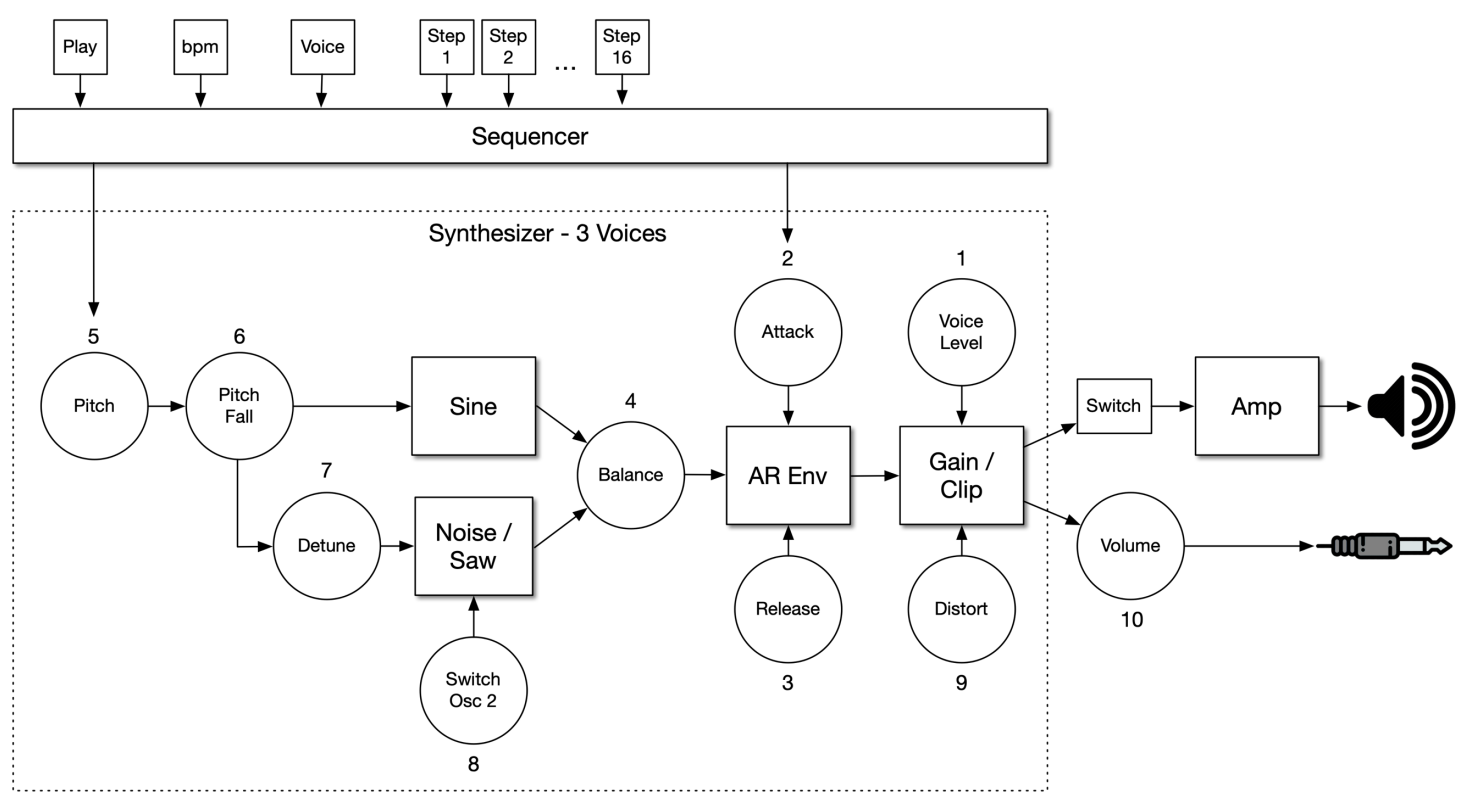

Fig. 2: The software architecture of Beat Machine 1.

Interactive systems, like the Beat Machine, are defined by their behaviour and temporal responsiveness, further underscoring the significance of feedback in relational interactions between users and the machine. The selection and responsiveness of interface controls and managing their update timing was an iterative process with many settings manually adjusted until the responsive 'felt' right. Constraints around the number of digital dials (eight) dictated synthesis parameter choices. Dials 9 and 10, for volume and distortion, were direct analog resistance controls (Fig 3). 
Brown \& Ferguson - Beat Machine, revised. 13 April 2020.

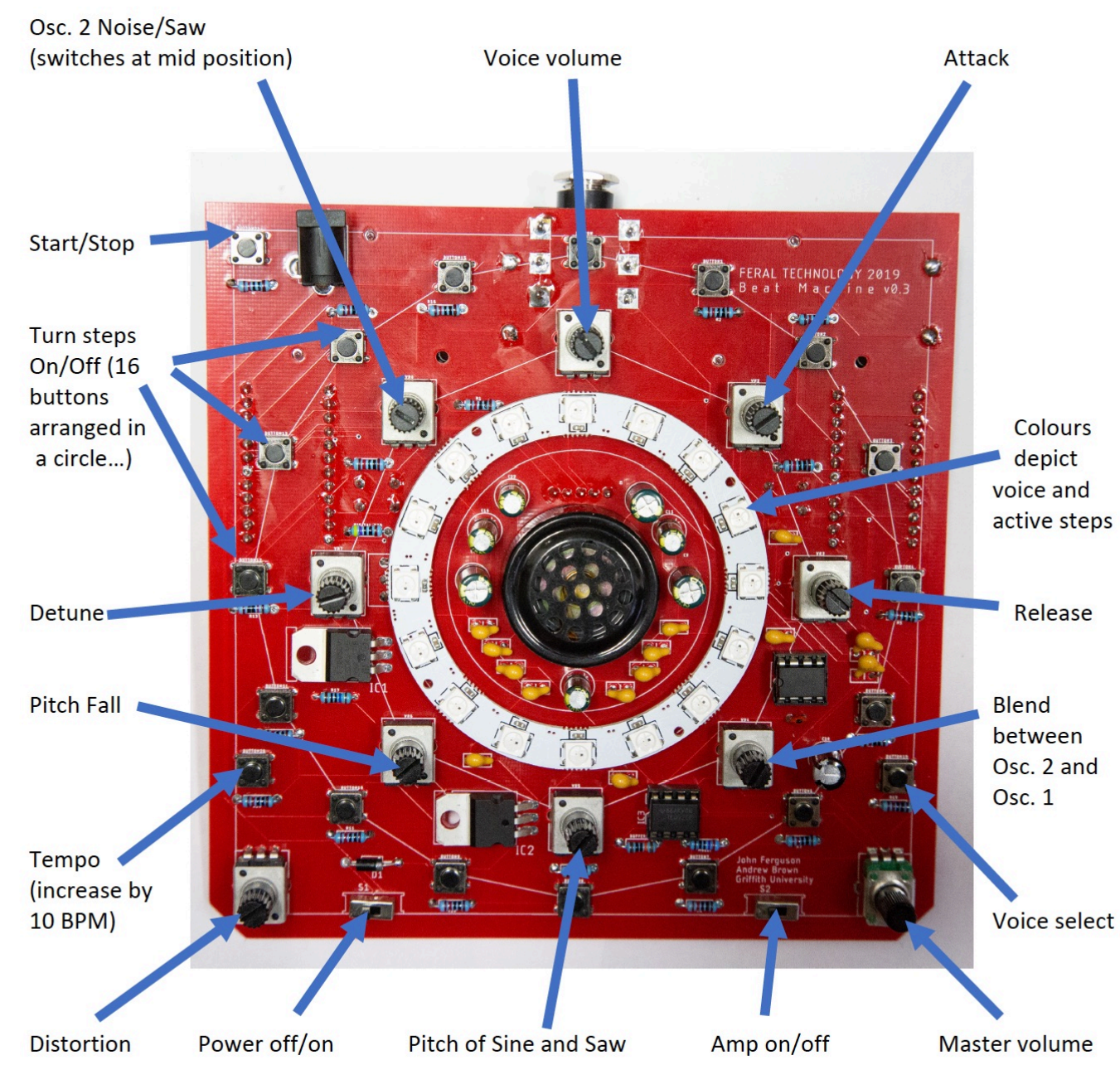

Fig. 3. Overview of Beat Machine controls

The Beat Machine's 16 step buttons, 8 dials, and control buttons exceeded the available GPIO pins on the Arduino. Additionally, the high-speed data communications protocol utilized by the neopixel to control LEDs had a tendency to cause havoc with the audio synthesis engine. The solution was to use a second microcontroller and two power regulators to minimise cross talk between sound generation and LED control. This approach also provided sufficient input pins for the desired level of control, communication between the two microcontrollers was made possible by use of the I2C interface. Another factor in this decision was that the 3 synthesis voices utilized all CPU capacity on the first Arduino, so sequencing, LED control and MIDI communication was allocated to the second Arduino. Software coordination between microcontrollers to manage distributed interface controls and coordinate timing of sequence and synthesis engines resulted in quite a tangle of code. But 8 
Brown \& Ferguson - Beat Machine, revised. 13 April 2020.

these forced design moves arose from the hardware affordances (or lack of them) and are further illustrative of the influence these components have in design decisions. Doing similar work on computing systems with significantly more power would not have made us so aware of each components' materiality.

The sequencing engine operates as a 16-step sequencer. It also incorporates generative features that enhance its presence in the musical production. These generative features are derived from the musical affordances of basic computational processes outlined by Brown and Sorensen [21]. In particular, they include the use of simple harmonic motion for imposing a groove accent, the ability of each step to have a probabilistic tendency to play or not, and each synthesis parameter can be adjusted per sequence step to allow either subtle or dramatic timbral variation from step to step.

The accelerometer activates when the device is held in excess of 15 degrees from horizontal and is currently configured as follows: Y (forward and back) controls the chance of adding random hits to the sequence; $X$ (left and right) effects the blend between Oscillator 2 and Oscillator 1 and can thus be used as a timbre control. Audio filtering for three voices was too computationally expensive to be effective on an Arduino, this approach to timbre using the $\mathrm{X}$ axis has proved a useful substitute.

The generative aspects of the system, though subtle, point toward a long-standing design tension between balancing reliance on the embodied skill of users (musicianship in this case) and ceding decision-making to algorithms or chance. This is relevant from an enactive perspective because such tension is based in an underlying dualist ontology of people and things. The enactive perspective tries to look past such human-machine asymmetries and the Beat Machine design allows for more or less generative influence in the music depending on user preference.

\section{System}

An important phase in the development of digital instruments such as the Beat Machine, is the collection of components and their integration. An enactive approach emphasises that this collection should include not only materials but deliberate decisions about social, cultural and environmental contributors to the design. During the Beat Machine development this 
Brown \& Ferguson - Beat Machine, revised. 13 April 2020.

included choices about target music genres and even musical works whose emulation would act as benchmarks for success. The establishment of a collection of technologies and concepts was ongoing and dynamic. Throughout the literature, such collections have been described as an "assemblage" [22] or "performance ecosystem" [23] or "technical milieu" [24]. Resources within such collections work best when they are complimentary in their contribution to the creative process [25]. The processes of enactive individuation arise through feedback on iterative product decisions which operate at multiple time scales. These processes reinforce or disrupt the technical/conceptual collections; decisions such as the Beat Machine's similarity to drum machines was made early on, decisions about hardware and software components and their integration accumulated as the project proceeded, whilst software choices could be more fluid and provisional.

Our creative development included interaction with layers of hardware and software built up over time. Figure 4 shows the development of Beat Machine version 3 at the stage of 'breadboard' design where most components had been selected and assembled to allow testing and refinement of the system. Software development typically followed hardware design with feedback processes revealing hardware constraints that required adaptation of features and occasional replacement of hardware elements.

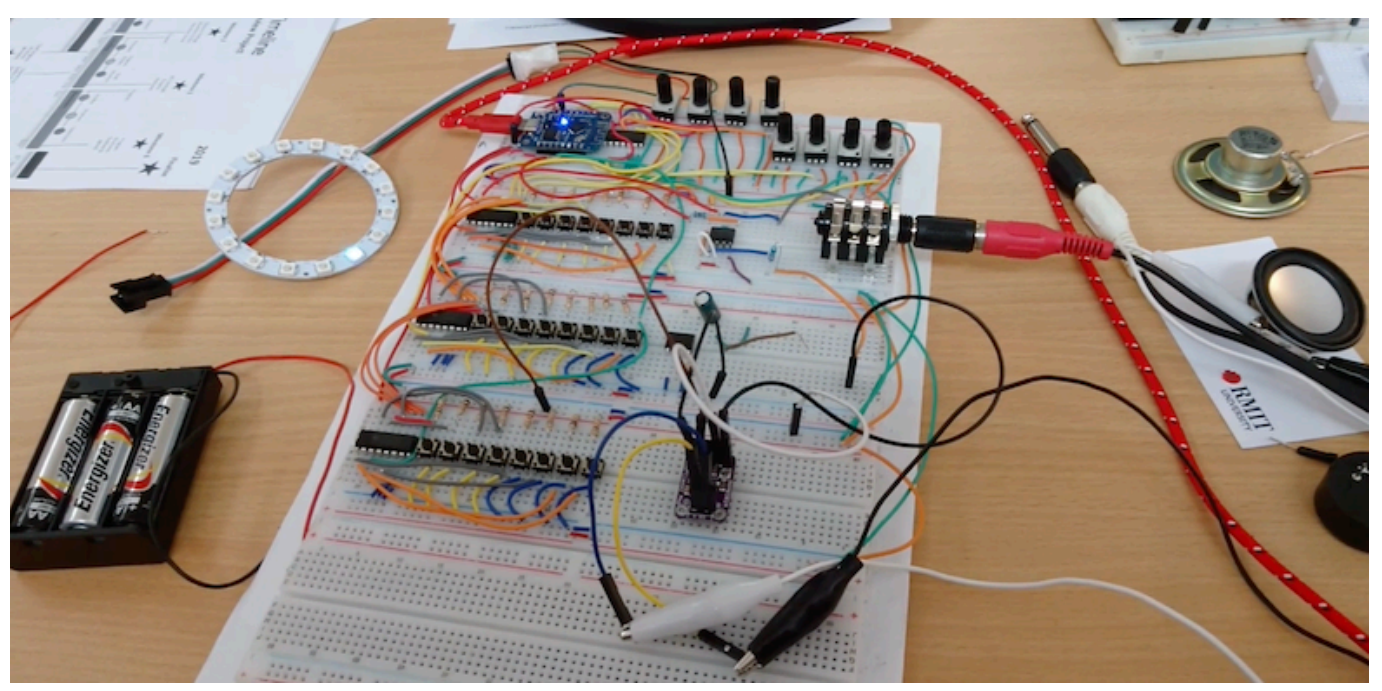

Fig. 4: Beat Machine version 3 during breadboard development (Wemos D1 and 4 x HC4051 Multiplexing chips). 
Brown \& Ferguson - Beat Machine, revised. 13 April 2020.

The entanglement of elements in the collection did at times become complex, however, it was often the solving of such wicked design problems that made the project interesting and satisfying.

Selected components including dual Arduino Nanos with regulated/isolated power supplies to minimise cross talk between LED control and sound generation, were laid out on a dedicated circuit board design shown in Figure 5. Bespoke circuit boards were produced by a commercial printing service in small quantities and components were hand soldered on by users.

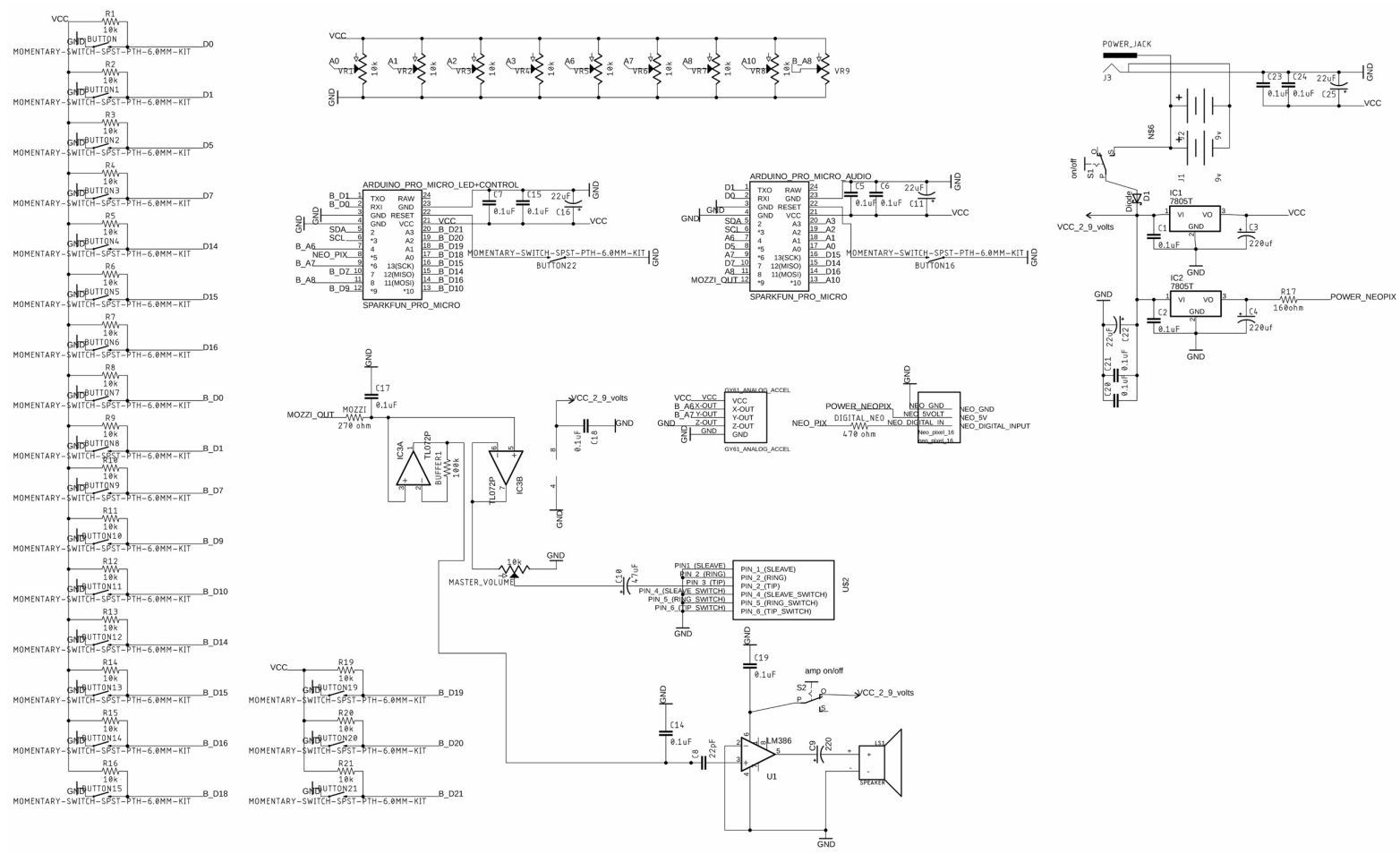

Fig. 5. Electronics Schematic created with EAGLE of Beat Machine version 1.

Throughout it all, the challenge was to work productively with materials that were simultaneously enabling and resistant. The individuality of the Beat Machine emerged from enactive engagement between these materials and our creative goals. Consistently, our experience was that solutions emerged through active interaction with the prototypes. 
Brown \& Ferguson - Beat Machine, revised. 13 April 2020.

\section{Conclusion}

The Beat Machine is a lo-fi musical groove box that relies on inexpensive and computationally limited hardware. In this project 'design' and 'making' are iterative activities full of learning, practising and skill development. As the project evolved we incrementally committed to hardware, software, and cultural elements; the design process congealed to form the particular individuality of the Beat Machine. In this way the project shares phenomenological features with other music practices, such as learning jazz improvisation described so insightfully by David Sudnow [26]. The project is shaped by material engagement with microelectronics in ways that fit neatly with the theory of enactive individuation.

The process of engaging with the materiality of electronic and digital components is visceral in a DIY project with such tight constraints, but we hope this case study serves to highlight how enactive individuation is a useful lens for understanding such a design process. As Poulsgaard articulates; "In a very real sense, technics forms the horizon of our imagination, our creative engagement with the world around us and our perception of temporality." [27] The challenge for us in analysing the Beat Machine project through the lens of enactive individuation was how to make sense of developmental ecologies that were at once material, informational, and cultural and which had the potential to shape our creative imagination and practice. In reflecting on our design and making activities we have embraced material engagement theories within a digital context. These may be seen as simple metaphoric projections from analog making processes. But through theoretical developments such as enactive individuation, the unique complexity of hybrid digital-physical practices, such as ours in making the Beat Machine, are increasingly recognised as authentic.

In this article, we've used reflections on the Beat Machine project to highlight how material engagement is at the heart of post-digital design and making practices. As musicians, we've found the concept of enactive individuation to be sympathetic with our commitment to a performative approach to creative interactions with materials and contexts. 
Brown \& Ferguson - Beat Machine, revised. 13 April 2020.

\section{References and Notes}

1. Nicholas Collins, Handmade Electronic Music: The Art of Hardware Hacking (New York: Routledge, 2006).

2. John Ferguson, "HST1d Feral Technologies" http://www.johnrobertferguson.com/hst1d-feral-technologies, (accessed December 30, 2019).

3. John Ferguson, "Beat Machine Introduction” https://vimeo.com/327839864, (accessed December 30, 2019).

4. Mihaly Csikszentmihalyi, Flow: The psychology of happiness (London: Rider Books, 1992).

5. Andrew R. Brown and Steve Dillon, "Meaningful Engagement with music composition," in The Act of Musical Composition: Studies in the creative process (Surrey, UK: Ashgate, 2012) pp. 79-110.

6. Marc Leman, "Musical homeostasis: a key concept for understanding humantechnology interactions," Socio-Cultural Role of Technology in Digital Musical Interactions, 2019, http://www.aalto.fi/en/events/socio-cultural-role-of-technology-indigital-musical-interactions, (accessed December 30, 2019).

7. Kim Cascone, "The Aesthetics of Failure: 'Post-digital' tendencies in contemporary computer music," Computer Music Journal 24, No. 4, 12-18 (2000).

8. John Ferguson and Andrew R. Brown, "Fostering a post-digital avant-garde: Research-led teaching of music technology," Organised Sound 21, No. 02, 127-137 (2016).

9. Kåre Stokholm Poulsgaard, "Enactive individuation: Technics, temporality and affect in digital design and fabrication," Phenomenology and the Cognitive Sciences 18, No. 1, 281-298 (2019).

10. Lambros Malafouris, "The cognitive basis of material engagement: where brain, body and culture conflate," in Rethinking materiality: The engagement of mind with the material world (Cambridge: McDonald Institute Monographs, 2004) pp. 53-61.

11. Poulsgaard [9] p. 294.

12. Bernard Stiegler, Technics and Time, 1: The fault of Epimetheus (Stanford: Stanford University Press, 1998). 
Brown \& Ferguson - Beat Machine, revised. 13 April 2020.

13. Gilbert Simondon, “Translation: 'Individuation of Perceptive Units and Signification' Chapter 1 of Psychic and Collective Individuation," Speculative Heresy, 2008 [1989], https://speculativeheresy.wordpress.com/2008/10/06/translation-chapter-1-ofsimondons-psychic-and-collective-individuation/, (accessed December 30, 2019).

14. Martin Heidegger, The Question Concerning Technology and Other Essays (New York: Harper \& Row, 1977).

15. Stiegler [12]

16. Poulsgaard [9] p. 288.

17. Heekyoung Jung, Heather Wiltse, Mikael Wiberg, and Erik Stolterman, "Metaphors, materialities, and affordances: Hybrid morphologies in the design of interactive artifacts," Design Studies 53, 24-46 (2017).

18. Jung et al. [17] p. 5.

19. Tim Barrass, "Mozzi: Audio synthesis library for Arduino.” (2012) https://sensorium.github.io/Mozzi/, (accessed December 30, 2019).

20. Poulsgaard [9] p. 287.

21. Andrew Sorensen and Andrew R. Brown, "aa-cell in practice: an approach to musical live coding," in Proceedings of the International Computer Music Conference (ICMA: Copenhagen, 2007) pp. 292-299.

22. Bruno Latour, “On Actor-Network Theory: A Few Clarifications," Soziale Welt 47, No. 4, 369-381 (1996).

23. Simon Waters, "Performance ecosystems: Ecological approaches to musical interaction," in Proceedings of the Electroacoustic Music Studies Network (EMS: Leicester, UK, 2007) pp. 1-20.

24. John Sutton, "Material agency, skills and history: Distributed cognition and the archaeology of memory," in Material Agency: Towards a Non-Anthropocentric Approach (Springer, 2008) pp. 37-55.

25. Edwin Hutchins, Cognition in the wild (Cambridge, MA: The MIT Press, 1995).

26. David Sudnow, Ways of the hand: The organization of improvised conduct (Cambridge, MA: The MIT Press, 1993).

27. Poulsgaard [9] p. 286. 
Brown \& Ferguson - Beat Machine, revised. 13 April 2020.

Biographical Information

Prof. Andrew R. Brown is Professor of Digital Arts at Griffith University in Brisbane, Australia.

Dr John R. Ferguson is Head of Creative Music Technology at Griffith University in Brisbane. 